\title{
Rising bubble in a cell with a high aspect ratio cross-section filled with a viscous fluid and its connection to viscous fingering
}

\author{
Mayuko Murano and Ko Okumura $\odot^{*}$ \\ Department of Physics and Soft Matter Center, Ochanomizu University, 2-1-1, Otsuka, Bunkyo-ku, Tokyo 112-8610, Japan
}

(Received 10 December 2018; accepted 10 December 2019; published 21 February 2020)

\begin{abstract}
We investigate, both experimentally and theoretically, the velocity and shape of bubbles that rise in a vertical cell with a rectangular cross-section due to buoyancy. The shorter length of the cross-section is comparable to, and the longer length is larger than, the capillary length. This geometry allows us to confine the bubble in two lateral directions, where the bubble is strongly confined by the front and back walls of the cell, and weakly confined by the side walls. Due to this double confinement, one lateral dimension of the bubble is comparable to, but the other lateral and vertical dimensions are larger than, the capillary length. This combination of length scales results in the distinct behavior described in the present study. Our focus here is on the dynamics in the viscous regime, in which buoyancy acts as the driving force for the vertical motion, which is opposed by viscous drag. We have successfully established simple laws for the velocity and shape of the doubly confined bubbles, which lead to a scaling law for the viscous drag acting on the bubble. It is shown that the downward flow around the bubble is essential to the dynamics in this regime. Confocal imaging on submillimeter scales reveals velocity profiles consistent with the present theory. We explore how this doubly confined regime exhibits crossover to previously known scaling laws, and present a corresponding phase diagram. By using a simple velocity transformation, we show the behavior of rising bubbles in the present study corresponds to, and leads to a deeper understanding of, certain types of viscous fingering.
\end{abstract}

DOI: 10.1103/PhysRevResearch.2.013188

\section{INTRODUCTION}

The dynamics of a bubble rising in a confined space is a classic problem that is replete with interesting physics yet to be explored. The bubble motion is driven by pressure, which typically originates from buoyancy or forced flow. This motion is opposed by viscous drag and/or inertia. The relevant dynamics are in the viscous regime when viscous drag dominates, and in the inertial regime when inertia dominates. When the length scales associated with confining space and/or bubbles are smaller than, or comparable to, the capillary length, capillarity becomes an important consideration. Because of the interplay between inertia, viscosity, capillarity, and gravity, this classic problem has continued to be an active area of research.

In this study, we focus on a viscous regime where the driving force for a bubble is a buoyant force that is opposed predominantly by viscous drag. There are several important length scales for the confining space and bubbles, and some of them are comparable to, and others are larger than, the capillary length. On the length scales considered in this work, capillarity effects are non-negligible in one lateral direction,

\footnotetext{
*okumura@phys.ocha.ac.jp

Published by the American Physical Society under the terms of the Creative Commons Attribution 4.0 International license. Further distribution of this work must maintain attribution to the author(s) and the published article's title, journal citation, and DOI.
}

in contrast to some studies [1]. The present study is also distinct from studies focusing on small rectangular tubes [2,3], where the length scales of confinement are smaller than the capillary length in both lateral directions. In our case, the rising velocity is inversely proportional to viscosity, and the capillary number $\mathrm{Ca}$ is smaller than unity ( $\mathrm{Ca}$ is typically from 0.01 to 0.1 ). Therefore the nonlinear viscous dynamics originating from meniscus dissipation is not dominant [4,5], as discussed below. However, in some cases, the meniscus dissipation becomes important for bubble dynamics [6].

Now, we will present a brief history of the study of rising bubbles in a confined space. Two pioneering studies were performed using two distinct geometries. One study by Saffman and Taylor in 1958 [7] used Hele-Shaw cells, and another by Bretherton in 1961 [8] used capillary tubes. In both studies, one of the main concerns was the viscous regime, which is the dominant regime in the present study. The length scales of the cross-section of the containers used by Bretherton tend to be smaller than the capillary length. However, those of Hele-Shaw cells used by Saffman and Taylor tend to be a mixture of length scales, comparable to (or smaller than), and larger than, the capillary length.

The Saffman-Taylor problem for the Hele-Shaw geometry [7] was revisited by Tanveer [9]. However, the theoretical models [7,9] were purely two-dimensional, implying that they did not consider the possibility that thin lubricating films exist between the bubble and the cell plates. Systematic experimental studies in a constant velocity regime were performed in a cell inclined to a nearly horizontal position [10-12]. In 2011 , bubble motion in a highly viscous liquid in a vertical 
cell was considered, to find a scaling regime in which viscous dissipation around the bubble is dominant, as in the case of Saffman and Taylor, but with the presence of a lubricating film [13]. (In this paper, another scaling regime was found for a viscous drop surrounded by a less viscous liquid.) It is also shown that the shape of the bubble in the viscous regime is almost circular [13], which is not always the case when inertia comes into play, as in the case of a bubble in water or in a moderately viscous oil [14]. In 2016, another scaling regime was found in which the dissipation in the lubricating film governs the dynamics, and the crossover of this regime to the scaling regime found in [13] was clearly shown [15] (see also the review paper [16]). In 2018, another scaling regime closely related to the one found in Ref. [15] was shown, and possible effects of long-range intermolecular interactions were discussed [17] (The role of the lubricating film and molecular interactions were discussed in the different context of oil droplets surrounded by water in microchannels in Ref. [18]). When inertia comes into play, the rising motion of a bubble tends to destabilize, but this is not the focus of the present study (see Ref. [14] for recent studies in this direction, which includes considerations of wake [19] and oscillation [20]). Numerical study including the existence of lubricating films is still a challenging problem, but this has been dealt with in the limited case in which the viscosity of the drop and the surrounding liquid are the same [21] (an empirical numerical treatment of the effect of dissipation in the lubricating film is discussed in Ref. [14]).

The capillary tube geometry of Bretherton is relevant to various problems such as air-liquid two-phase flow and, in particular, slug flow (see Refs. [22,23] for details), which has attracted considerable attention by virtue of its numerous practical applications. Gas-liquid slug flow is ubiquitous in industrial facilities, such as vapor generators, reboilers, buoyancy-driven fermenters, vaporizers, in the emergency core cooling of nuclear reactors, in oil extraction from wells [24], in transport of hydrocarbons in pipelines, and in geothermal and thermal power plants [25]. Accordingly, there are many fundamental studies on the rising dynamics of a single bubble in a tube with a circular cross-section [26-30]. With the development of microfluidics [31,32], many studies have been performed on the transportation of strongly confined bubbles in a tube with a square or rectangular cross-section with an aspect ratio close to one, both in the inertial [1,33-36], and viscous $[2,3,35,37-39]$ regimes. However, in the context of microfluidics, the length scales of bubble confinement are smaller than the capillary length, which differentiates these studies from the present work.

Reviewing the literature to date, we asked what would happen if a vertically rising bubble was strongly confined in one direction, as in a Hele-Shaw cell, and weakly confined in a second direction, by using a vertical tube with a high aspect ratio rectangular cross-section. This question was briefly addressed in the viscous regime by Clanet et al. in 2004 [1]. They used tubes with large cross-sections in which the capillary effect was suppressed. In addition, their theoretical arguments on the topic were purely dimensional, with no discussion of the physical importance of liquid flow around the bubble.

In this study, we focus on the viscous dynamics of a bubble in a cell with a rectangular cross-section where the short sides (a) doubly confined case

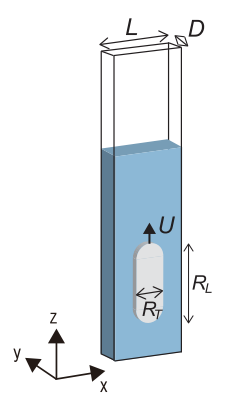

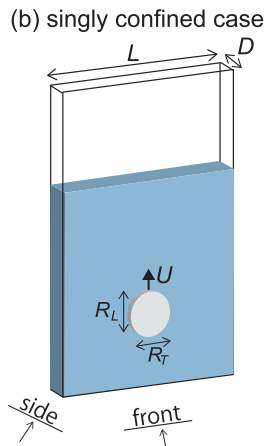

(c) side view

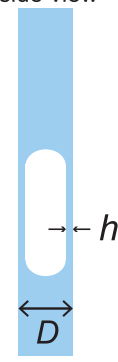

FIG. 1. Experimental setup for (a) the doubly confined regime ( $L$ is small) and (b) the singly confined regime ( $L$ is large). (c) Side view of a rising bubble.

are comparable to the capillary length, and long sides are much larger. The bubble volume is tuned so that the short sides strongly confine, and the long sides weakly confine the bubble. We then find simple laws for the velocity and shape of a bubble in mathematically closed forms, in which capillarity comes into play. In particular, we elucidate the importance of the conservation of liquid flow in determining the bubble's velocity, and how considering the downward flow around the bubble is essential. In addition, the velocity profiles of the derived expressions are directly confirmed by confocal imaging on submillimeter scales. To clarify the relationship of the present work to previous studies on the pure Hele-Shaw geometry [13], and to the case where there is no influence of capillarity [1], we demonstrate the crossovers to these regimes, and provide a phase diagram. Finally, we show that bubble rising behavior in the present study can be mapped to a type of viscous fingering using a velocity transformation, which provides novel insight into the study.

\section{EXPERIMENT}

As illustrated in Figs. 1(a) and 1(b), experiments were performed in a cell with a rectangular cross-section, which is made of transparent acrylic plates. The thickness $D$ and width $L$ are varied in a range of 0.5 to $3.0 \mathrm{~mm}$ and a range of 5 to $135 \mathrm{~mm}$, respectively, where $D \ll L$ is satisfied, i.e., the aspect ratio is high. We fill the cell with silicon oil of viscosity $\eta$, density $\rho$, and surface tension $\gamma(\eta=48.3-965 \mathrm{mPa} \mathrm{s}$, $\rho=960 \sim 970 \mathrm{~kg} / \mathrm{m}^{3}, \gamma=20 \mathrm{mN} / \mathrm{m}$ ) and inject air to make a bubble from the bottom of the cell. The bubble slowly rises at a constant speed. We investigate the rising velocity and shape of the bubble (see movie 1 and movie 2 [40]). As illustrated in Fig. 1(c), a liquid thin film of thickness $h$ exists between the bubble and a cell wall (see below for more details).

To characterize the dynamics, we introduce the characteristic radius of the disk-shaped bubble $r$ defined by $\pi r^{2} D=\Omega$ for the bubble volume $\Omega$ :

$$
r=\sqrt{\Omega / \pi D} .
$$

We introduce "the singly confined case" and "the doubly confined case," and put more focus on the latter. In both cases, the bubble is confined in the direction of the cell thickness $D$ : 

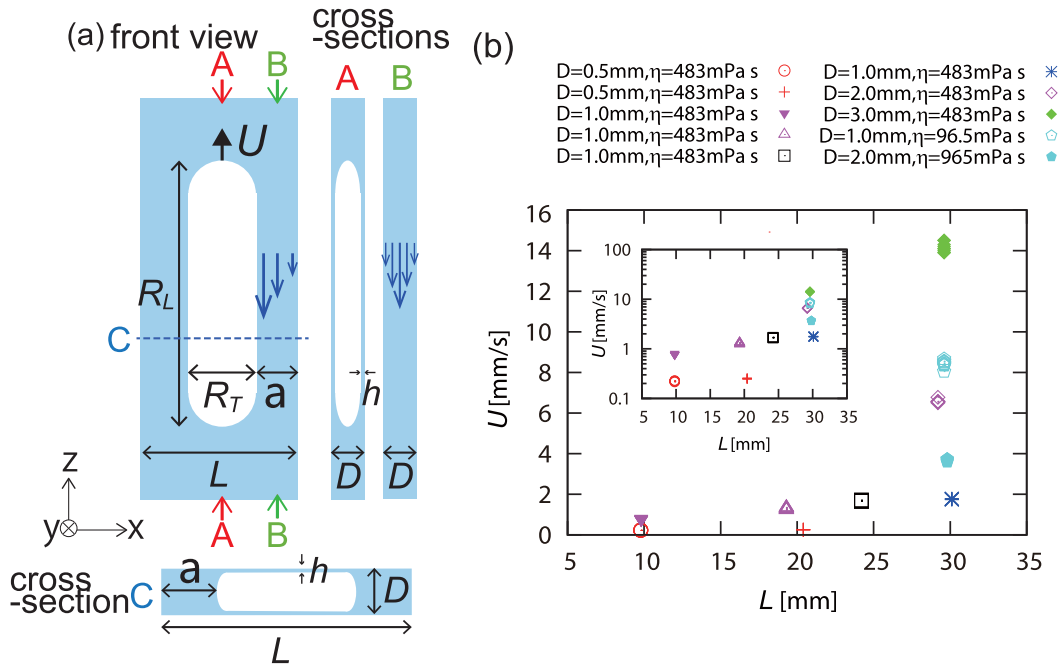

(c)
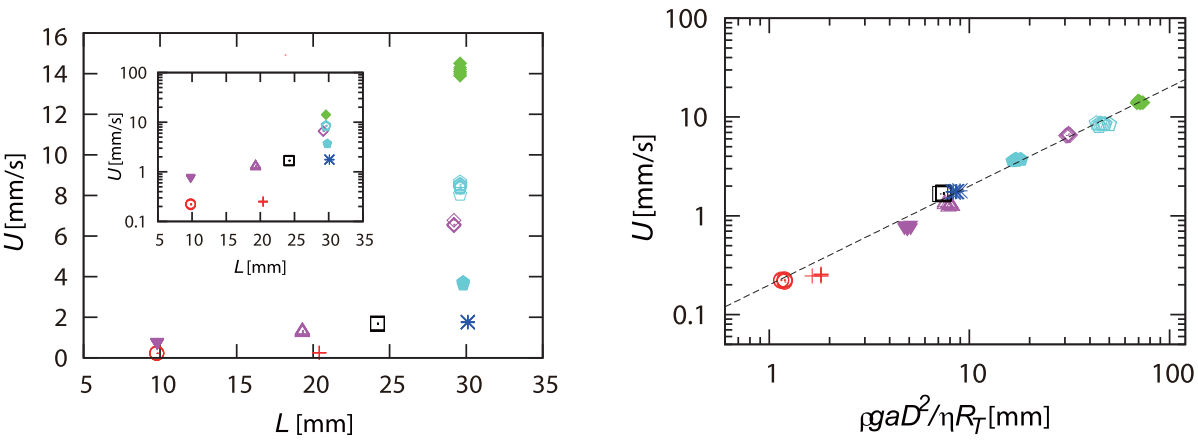

FIG. 2. (a) Rising bubble in the doubly confined regime. Cross-sections at the places indicated by two pairs of arrows (A, B) or a dashed line (C) in the front view are shown on the right-hand side (A, B) and at the bottom (C) of the front view. (b) Rising velocity $U$ as a function of cell width $L$ for various cell thickness $D$ and oil viscosity $\eta$. (Inset) Semilogarithmic plot of the same data. (c) Rising velocity $U$ vs $\eta g a D^{2} /\left(\eta R_{T}\right)$ on a log-log scale. Dashed line corresponds to Eq. (8) with $k_{1}=0.196 \pm 0.0015$.

the condition $D \ll 2 r$ is always satisfied (in addition to the above-mentioned condition $D \ll L$ for the cell geometry). In the singly and doubly confined cases, $L$ is significantly larger and smaller than $2 r$, respectively. The singly confined case corresponds to experiments using Hele-Shaw cells [13,1517]. In summary, in this paper, we distinguish the following two cases as illustrated in Figs. 1(a) and 1(b) and focus on the latter:

$$
\begin{aligned}
& L \gg 2 r \gg D \text { ( singly confined), } \\
& 2 r \gg L \gg D \text { (doubly confined). }
\end{aligned}
$$

In the present experimental study, the capillary length $\kappa^{-1}=$ $\sqrt{\gamma /(\rho g)}$ with $g$ the gravitational acceleration is roughly close to the cell thickness $D$ although it can be larger or smaller than $D$ (in experiment, it was in the range $0.5-3 \mathrm{~mm}$, as mentioned before):

$$
D \approx \kappa^{-1}
$$

Equation (4), together with Eqs. (2) and (3) implies that $L$ is larger than the capillary length (in experiment, it was in the range 5-135 $\mathrm{mm}$, as mentioned before). In other words, our study presented below is in contrast with studies such as [2] that focused on capillary tubes whose cross-section is characterized by length scales which are all smaller than the capillary length and, thus, neglected the gravity term in the Navier-Stokes equation.

\section{RESULTS}

\section{A. Rising velocity in the doubly confined regime}

In the viscous regime, the rising velocity $U$ can be derived from a balance between the gravitational and dissipative energies. The rising motion is driven by the decrease in a gravitational energy, which is dimensionally estimated as $\rho g R_{T} R_{L} D U$ per unit time, where $R_{T}$ and $R_{L}$ are the width and length of bubbles, respectively, as shown in Fig. 2(a).

The viscous dissipation is mainly written in the following four forms (dissipation in the dynamic meniscus is discussed later). First two types of dissipation occur in the regions outside of a bubble around the top and rear of the bubble. They are estimated as $T \dot{S}_{1} \cong \eta\left(U / R_{T}\right)^{2} R_{T}^{2} D$, which is caused by the velocity gradient in the $x$ direction $\left(\sim U / R_{T}\right)$, and $T \dot{S}_{2} \cong \eta(U / D)^{2} R_{T}^{2} D$, which is caused by the velocity gradient in the $y$ direction $(\sim U / D)$. [See Fig. 1(a) for the definitions of the $x$ and $y$ directions.]

The other two types of dissipation occur at the lateral sides of the bubble. As the bubble rises, downward flows are generated in the two gaps of width $a$ between side walls and the bubble (see movie 2 [40]):

$$
a=\frac{L-R_{T}}{2} .
$$

The average velocity of the flow $\bar{u}$ is determined by volume conservation:

$$
R_{T} D U=2 a D \bar{u} .
$$

Velocity gradients in the $x$ direction $\bar{u} / a$ and in the $y$ direction $\bar{u} / D$ (see Sec. III D for the experimental validation of the existence of these velocity gradients) cause the energy dissipations $T \dot{S}_{3} \cong \eta(\bar{u} / a)^{2} a R_{L} D$ and $T \dot{S}_{4} \cong \eta(\bar{u} / D)^{2} a R_{L} D$, respectively.

Since the oil totally wets the cell plate, two thin films of thickness $h$ exist between a bubble and cell plates. More specifically, for example, one of the films develops between the front surface of a bubble and the back surface of the front cell wall. These films are visible and dewetting of the thin film can not be recognized. The measurement of $h$ is in general difficult as discussed in Ref. [15], because several reflections are involved although the thickness is typically on submillimeter scales. Inside these thin films, no flow is developed in the present case. This was pointed out for a rising bubble in Ref. [13], and the necessary condition in a 
(a)

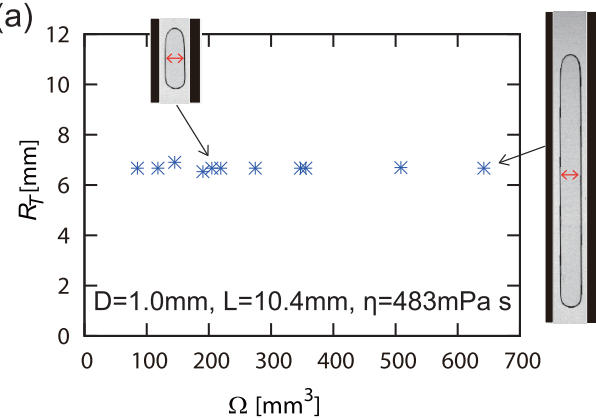

(c) 3

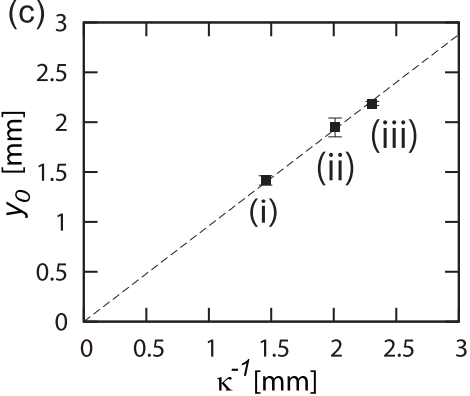

(b) Doubly confined case
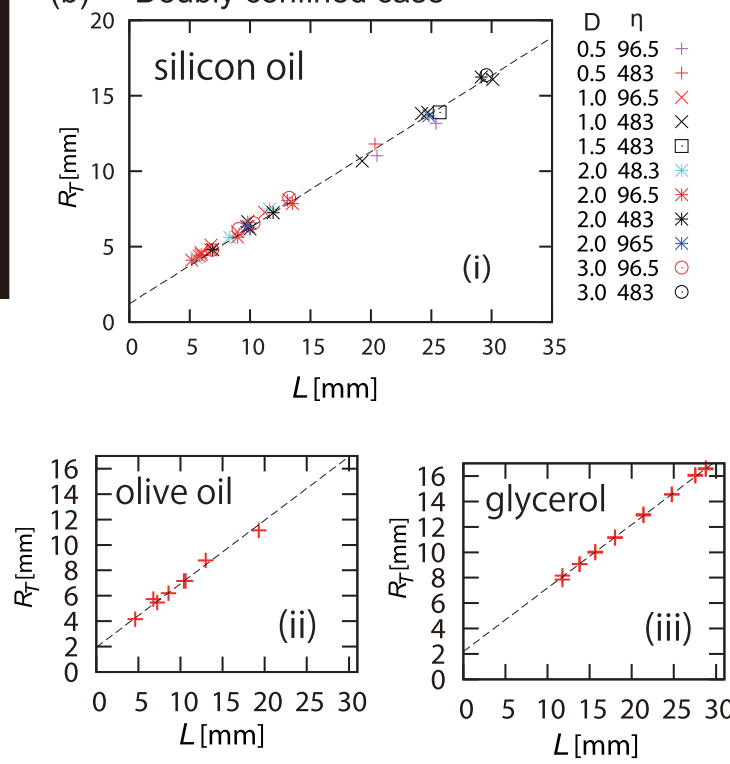

FIG. 3. (a) $R_{T}$ vs $\Omega$ for fixed $D, L$, and $\eta$. (b) $R_{T}$ vs $L$ for three different liquids. $R_{T}$ increases linearly with $L$ and depends on neither $D$ nor $\eta$. $D$ and $\eta$ are given in mm and mPa s, respectively. The dotted lines correspond to $R_{T}=L / 2+y_{0}$ where $y_{0}$ is determined by fitting. (c) Capillary length of each liquid $\kappa^{-1}$ vs $y_{0}$ obtained in (b), showing the relation $y_{0} \cong \kappa^{-1}$.

more general case in which an air bubble could be replaced with a fluid bubble was established in Refs. $[15,16]$. The underlining physics is the competition between two extreme cases: one case in which the flow velocity gradient develops only inside a fluid drop and the other in which it develops only inside the thin liquid films. In the former extreme, the flow inside the thin films is suppressed, which is possible because a downward flow inside the fluid drop can cancel out the velocity at the liquid-fluid interface defining the surface of the fluid drop.

When Eq. (3) is "strongly satisfied" under Eq. (4) in a sense that $L \gg D$ in Eq. (3) can be replaced with

$$
L \gg 4 D+2 \kappa^{-1},
$$

$T \dot{S}_{4}$ dominates over the other three, as explained in Sec. IV B 1. Under this strong double confinement, from the balance of this dominant dissipative energy and the gravitational one, i.e., $\rho g R_{T} R_{L} D U \cong \eta(\bar{u} / D)^{2} a R_{L} D$ with the aid of Eq. (6), we obtain the following rising velocity:

$$
U=k_{1} \frac{\rho g}{\eta} \frac{a}{R_{T}} D^{2} \text { (doubly confined) }
$$

with a numerical coefficient $k_{1}$ to be determined experimentally. In the following, when discussing velocity, we assume the condition of the strong double confinement is satisfied in the doubly confined case.

As shown in Figs. 2(b) and 2(c), Eq. (8) agrees well with experiment. The results of the measurement of $U$ as a function of $L$ for various $D$ and $\eta$ satisfying Eqs. (3) and (7) are shown in Fig. 2(b). The same data are replotted, with horizontal axis renormalized according to Eq. (8), in Fig. 2(c), in which a clear collapse of the data is demonstrated with $k_{1}=0.196 \pm$ 0.0015. In Fig. 2(c), we used experimentally measured value of $a$ given in Eq. (5), although we give an expression for $R_{T}$ in Eq. (9) below. The reason will be explained in Sec. III C.

We did not consider the viscous dissipation in the dynamic meniscus in the above, which is justified as follows. It is known that the corresponding viscous dissipation results in nonlinearity. This is because the thickness of the dynamic meniscus is comparable to the thickness $h$ discussed above, which is nonlinearly dependent on the capillary number defined as $\mathrm{Ca}=\eta V / \gamma$ when $\mathrm{Ca}$ is not much larger than one, which is the present case: $h \simeq \kappa^{-1} \mathrm{Ca}^{2 / 3}$ for $\mathrm{Ca} \ll 1$ and $h \simeq$ $\kappa^{-1} \mathrm{Ca}^{1 / 2}$ for $\mathrm{Ca} \simeq 1[4,5]$. The dissipation in the dynamic meniscus per unit time scales as $\eta(V / h)^{2}$ multiplied by the volume of the meniscus. The driving force in the present case is the buoyant force and is independent of viscosity. Thus the balance of the dissipation force and the driving force results in the rising velocity that is not inversely proportional $\eta$. On the contrary, our measured velocities are clearly inversely proportional to viscosity as seen above, which justifies ruling out the possibility that the dissipation in the dynamic meniscus is predominant.

\section{B. Bubble width in the doubly confined regime}

We here discuss how $R_{T}$, which characterizes the bubble shape, is selected for a rising bubble. As seen below we confirm experimentally the following relation for $R_{T}$ in the confined regime, in which the volume of the bubble $\Omega$ is large:

$$
R_{T}(\Omega)=\frac{1}{2} L+\kappa^{-1} \text { (doubly confined). }
$$

This relation, which holds up to the numerical coefficients, is shown in the following three steps. First, as shown in Fig. 3(a), for fixed $L, D$, and $\eta$, the transverse bubble size $R_{T}$ is independent of $\Omega$. Second, three plots in Fig. 3(b) show that $R_{T}$ linearly increases with $L$, with a slope $1 / 2$ and 
that $R_{T}$ is independent of $D$ and $\eta$. This is shown for three liquids, PDMS, olive oil $\left(\gamma=32 \mathrm{mN} / \mathrm{m}, \rho=914 \mathrm{~kg} / \mathrm{m}^{3}\right)$ and glycerol $\left(\gamma=63.4 \mathrm{mN} / \mathrm{m}, \rho=1260 \mathrm{~kg} / \mathrm{m}^{3}\right)$. Finally, we determine $y_{0}$ in Fig. 3(b) as the intercept of an extrapolated line with the vertical axis by fitting the data with $R_{T}=$ $1 / 2 L+y_{0}$ and show that $y_{0} \cong \kappa^{-1}$ as follows. In Fig. 3(c), the vertical axis is $y_{0}$ extracted from Fig. 3(b) as above, and the horizontal axis is the capillary length of each liquid, which are calculated from the definition $\kappa^{-1}=\sqrt{\gamma /(\rho g)}$ (the calculated values are $\kappa^{-1}=1.45,2.01$, and $2.31 \mathrm{~mm}$ for PDMS, olive oil, and glycerol, respectively). The straight line that fits the three data points in Fig. 3(c) establishes the relation $y_{0} \cong \kappa^{-1}$ and, thus, Eq. (9).

Equation (9) is satisfied even if Eq. (7) is not satisfied as long as Eqs. (3) and (4) are satisfied. In fact, Fig. 3(b) contains such cases, in which $a$ is comparable to, but is not less than, the capillary length. In the present study, $a$ is typically larger than, and is even in the smallest case comparable to, the capillary length.

However, since $a$ could be of the order of the capillary length as $D$ is, we need to clarify our definition of $R_{T}$, or equivalently, how we determined $a$. In the present study, we measured the outer edge of a bubble, corresponding to the tip of menisci to define $R_{T}$.

In microfluidics, a bubble is frequently confined strongly in a rectangular capillary tube whose short and long sides of the cross-section are both smaller than the capillary length. In such a case, the confined bubble tends to touch walls through thin lubricating films, making menisci at the four corners [2,3]. The thickness $h$ of such lubricating films is much smaller than the capillary length. This is in contrast with the present case, because $h$ corresponds to $a$ in the strongly confined microfluidics case: Eq. (9) is replaced with $R_{T}=L-2 h$, i.e., $h=\left(L-R_{T}\right) / 2$, which parallels Eq. (5).

Equation (9) is analogues to a result known for a viscous finger created in a Hele-Shaw cell. The finger width has been experimentally shown to be the half of the cell width, $L / 2$ (instead of $L / 2+\kappa^{-1}$ ), for a cell of width $L$, when the capillary number is not too small. The reason was theoretically explained in Ref. [41]. However, it is possible that the precision of the previous experiments cannot distinguish the difference between $L / 2$ and $L / 2+\kappa^{-1}$ (see Sec. V for more details).

\section{Closed form for the velocity and drag friction}

From Eqs. (8) and (9), the rising velocity in the doubly confined regime can be expressed using only experimentally controllable parameters, i.e., without using the quantity $R_{T}$ [note that $R_{T}$ in Eq. (8) cannot be controlled experimentally, if we do not know Eq. (9)]:

$$
U=\frac{k_{1}}{2} \frac{\rho g}{\eta} \frac{L-2 \kappa^{-1}}{L+2 \kappa^{-1}} D^{2} \text { (doubly confined). }
$$

We explain the reason we used experimentally measured value of $a=\left(L-R_{T}\right) / 2$ in Fig. 2(c), as announced. Equation (8) can be explained theoretically (with treating $a$ or $R_{T}$ as an experimentally given quantity) and can be confirmed experimentally, whereas Eq. (9) cannot be explained theoretically but can be confirmed experimentally in a rather clear manner. The two confirmations presented in the above have clear meanings: one for the confirmation of a theoretical consideration leading to Eq. (8) and the other for the confirmation of the experimentally emerged relation in Eq. (9). If we provided instead a single confirmation of Eq. (10) [using the $R_{T}$ value from Fig. 3 and Eq. (9)], the meaning of confirmation becomes less clear.

We can now derive the viscous drag friction acting on a bubble in the doubly confined regime, an generalized formula of the well-known Stokes' drag friction. (For other forms of generalization in different regimes, see Refs. $[13,15,16]$.) In Sec. III A, the rising velocity in Eq. (8) is obtained by an energy balance. The same velocity should be reproduced from the force balance $F_{g}=F_{\eta}$, where $F_{g}=\rho g \Omega$ is the gravitational force and $F_{\eta}$ is the drag force in question. From this balance, with the aid of Eq. (10), we readily obtain the following drag force acting on a rising bubble:

$$
F_{\eta}=\frac{2}{k_{1}} \eta \frac{\Omega\left(L+2 \kappa^{-1}\right)}{D^{2}\left(L-2 \kappa^{-1}\right)} U \text { (doubly confined). }
$$

\section{Velocity profile inside the cell by Particle tracking velocimetry (PTV)}

Particles (techpolymer MBX-20, SEKISUI PLASTICS) are added into the oil to visualize the flow in the gap between the rising bubble and the side walls to justify the assumption we made in obtaining the scaling laws. We use a microscope lens $($ PLN10 $\times$ or PLN4 $\times$, OLYMPUS) such that the depth of the focus in the magnified movie is approximately $50-100 \mu \mathrm{m}$. We measure the velocity by tracking particles at different distances between the cell and camera using an optical stage equipped with a micrometer (BXT06013C, Surugaseiki, Japan), which allows confocal imaging on submillimeter scales. The density and diameter of the particles are respectively given by $\rho_{b}=1.20 \mathrm{~g} / \mathrm{cm}^{3}$ and $d=20 \mu \mathrm{m}$. Although the density is not perfectly matched with that of the oil, this does not affect the precision of the PTV measurement. This is because the descending velocity of the small particles, scaling as $\left(\rho_{b}-\rho\right) g d^{2} / \eta$ with $\rho$ and $\eta$ the density and viscosity of the oil, is much smaller than a typical velocity of the measured flow.

Figure 4(a) shows the velocity of particles as a function of the distance from the side wall $\Delta x$ in the presence of a bubble. Different colors of markers indicate the velocity of particles at different distance from the front wall $\Delta y$. The profile of the flow in the gap shown in this plot is qualitatively consistent with a Poiseuille flow developed over the distance $a$ ( $a=$ $1.57 \mathrm{~mm}$ ) with the boundary conditions, zero velocity at the side wall and zero velocity gradient at the free surface of the bubble $(\Delta x \cong 1.6 \mathrm{~mm})$. The latter condition is expected because the liquid does not contain any surfactants. Figure 4(b) shows the velocity of particles in the region outside of the bubble. The profiles are given at the distance $\Delta x=1.2,0.5$, and $0.3 \mathrm{~mm}$ from the side wall, as a function of the distance from the front wall $\Delta y$. The flow profile shown in the plot is again qualitatively consistent with a Poiseuille flow developed over the distance $D(D=1.5 \mathrm{~mm})$.

For a technical reason, we selected the case of relatively large $D$ and fairly small $a$ for the confocal analysis. This case 
(a)

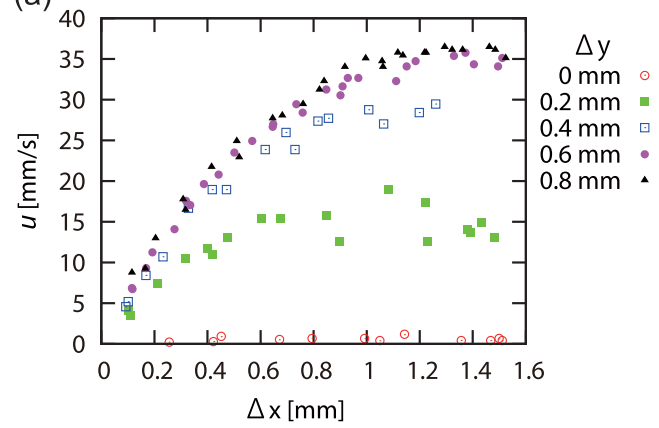

(b)

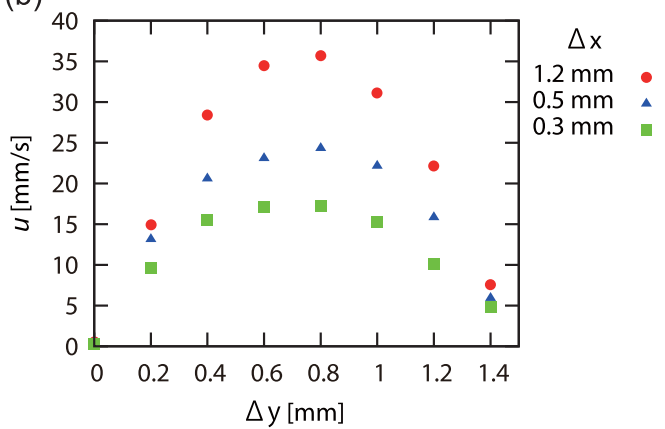

FIG. 4. (a) Flow plofile over the distance $a(=1.57 \mathrm{~mm})$ : particle velocity vs. distance from the side wall at the depth $\Delta y=0.2,0.4,0.6$, and $0.8 \mathrm{~mm}(L=8.96 \mathrm{~mm}, D=1.5 \mathrm{~mm}$, and $\eta=0.098 \mathrm{mPa} \mathrm{s})$ (b) Flow plofile over the distance $D(=1.5 \mathrm{~mm})$ : particle velocity vs. depth from the front wall at the distance $\Delta x=1.2,0.5$, and $0.3 \mathrm{~mm}$ from the side wall.

does not satisfy Eq. (7), equivalent to the relation $D \ll a$ in eq. (15) below. The data obtained in this case was not categorized as the one in the doubly confined regime when discussing velocity. For example, the corresponding data was not plotted in Fig. 2(b), which collect the velocity data in the doubly confined regime. Nonetheless, Fig. 4 supports our theory. This is because our theory assumes the existence of the above two types of Poiseuille flows, irrespective of magnitude relation between $D$ and $a$, and this assumption is consistent with Fig. 4.

\section{RELATIONS TO OTHER DYNAMIC REGIMES}

\section{A. Small-capillarity limit of the doubly confined regime}

Our velocity law given in Eq. (10) generalizes and thus contains a previous result established in Ref. [1] as shown below. Equation (10) can be recast into the following form

$$
U=\frac{k_{1}}{2} \frac{\rho g}{\eta} D^{2} \frac{1-2 \sqrt{1 / B o}}{1+2 \sqrt{1 / B o}} \text { (doubly confined) }
$$

by introducing Bond number:

$$
\mathrm{Bo}=\rho g L^{2} / \gamma \text {. }
$$

In the large Bo limit of small capillarity effect, Eq. (12) becomes

$$
U=\frac{k_{1}}{2} \frac{\rho g}{\eta} D^{2} .
$$

This expression was found to describe the viscous regime of the bubble rising in tubes with large rectangular cross-sections when the aspect ratio becomes large but the bubble is still confined from two directions $(L<r)$ [1]. In Ref. [1], Eq. (14) is confirmed for Bo $\sim 10^{4}$, which is much larger than values of $\operatorname{Bo}\left(\sim 10^{2}\right)$ in Fig. 2.

\section{B. Crossover of rising dynamics to the singly confined regime}

We next consider the crossover of the velocity and bubble shape to the singly confined regime.

\section{Velocity crossover}

We now justify the velocity law given in Eq. (8) in the doubly confined regime, in which Eqs. (3) and (7) are valid, by showing the relation $T \dot{S}_{4} \gg T \dot{S}_{3}, T \dot{S}_{2}$, and $T \dot{S}_{1}$, which respectively correspond to

$$
D \ll a, \quad a \ll R_{L}, \quad\left(D / R_{T}\right)^{2} \ll R_{L} / a .
$$

The first relation is satisfied because this is equivalent to Eq. (7). To examine the second relation, we note that, from the condition $L \ll 2 r$ in Eq. (3), the bubble is generally elongated:

$$
R_{L} \gg 2 r \gg R_{T} \text {. }
$$

From Eq. (5), $a<L / 2$ (because $R_{T}>0$ ), which means $a<$ $L \ll 2 r \ll R_{L}$ and thus proves the second relation in Eq. (15), $a \ll R_{L}$. This last relation tells that the third relation in Eq. (15) is satisfied if $D<R_{T}$, which can be checked from $D \ll a$, with the aid of Eq. (9) found to be valid experimentally: $a=L / 4-\kappa^{-1} / 2<R_{T}=L / 2+\kappa^{-1}$. The above arguments lead to the relation $D \ll a<R_{T}<L \ll 2 r \ll R_{L}$, where $R_{T}<L$ comes from the condition $a>0$.

On the contrary, in the singly confined limit, as seen in Fig. 5(b) below, we have the following approximate relation [instead of Eq. (9)]:

$$
R_{T} \approx R_{L} \approx 2 r \text { (singly confined). }
$$

Since $L \gg 2 r$ in the singly confined limit, this means that $a$ is of the order of $L$, and we see that $a \gg R_{L}$, for which $T \dot{S}_{2} \gg T \dot{S}_{4}$, while we have $D \ll a\left(T \dot{S}_{3} \ll T \dot{S}_{4}\right.$ and thus $\left.T \dot{S}_{2} \gg T \dot{S}_{3}\right)$ and $D \ll R_{T}\left(T \dot{S}_{1} \ll T \dot{S}_{2}\right)$ from Eq. (2), i.e., $T \dot{S}_{2} \gg T \dot{S}_{4}, T \dot{S}_{3}$, and $T \dot{S}_{1}$. In other words, in the singly confined regime, from the balance between the gravitational energy $\rho g R_{T} R_{L} D U$ and the dissipation energy $T \dot{S}_{2}$, we obtain

$$
U=k_{2} \frac{\rho g}{\eta} \frac{R_{L}}{R_{T}} D^{2} \text { (singly confined). }
$$

This is experimentally confirmed in Ref. [13] even under the existence of a lubricating film with coefficient $k_{2} \cong 0.08$. Note here that in the singly confined regime we have

$$
2 r \gg \kappa^{-1} \text {. }
$$

This is because $R_{T} \gg D$ is required for $T \dot{S}_{1} \ll T \dot{S}_{2}$ to be valid, while $R_{T} \approx r$ and $D \approx \kappa^{-1}$. 

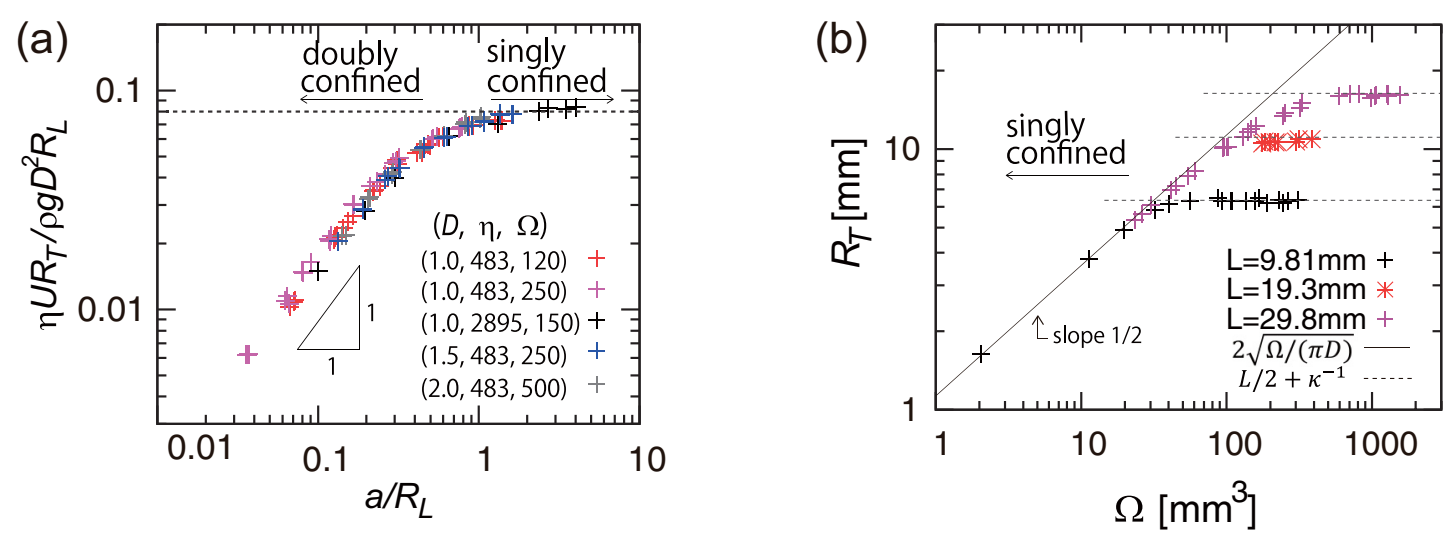

FIG. 5. (a) Renormalized velocity $U$ vs $a / R_{L}$ on a log-log scale, which shows the velocity crossover. The dotted line corresponds to $\frac{\eta U R_{T}}{\rho g R_{L} D^{2}}=k_{2}$ (here, taken to be 0.08 as established in Ref. [13].) $D, \eta$, and $\Omega$ are given in the units mm, mPa s, and mm ${ }^{3}$, respectively. (b) Bubble width $R_{T}$ vs volume $\Omega(D=1.0 \mathrm{~mm}$ and $\eta=483 \mathrm{mPa}$ s) for different cell width $L$, which shows the bubble-shape crossover. The cross-point of the line with slope $1 / 2$ with the horizontal line for each $L$ defines the crossover volume $r^{2} D \sim\left(L / 2+\kappa^{-1}\right)^{2} D($ or $r \sim L$ for $\left.L>\kappa^{-1}\right)$.

It is convenient to recast the velocity laws in Eqs. (8) and (18) into the following dimensionless form:

$$
\frac{\eta U R_{T}}{\rho g R_{L} D^{2}}=\left\{\begin{array}{ll}
k_{1} a / R_{L} & \text { (doubly confined: } \left.a / R_{L} \ll 1\right) \\
k_{2} & \text { (singly confined: } \left.a / R_{L} \gg 1\right)
\end{array}\right. \text {. }
$$

This is because, as seen above, important relations differentiating the velocity laws in the doubly and singly confined regime are the following: under Eq. (3), $r \gg L \Leftrightarrow a / R_{L} \ll 1$ while, under Eq. (2), $r \ll L \Leftrightarrow a / R_{L} \gg 1$.

Figure 5(a) shows the relation between $\eta U R_{T} /\left(\rho g R_{L} D^{2}\right)$ and $a / R_{L}$ on a log-log scale, which clearly shows the crossover predicted in Eq. (20). In order to confirm this crossover from the doubly to singly confined regime, we experimentally change the ratio of $a$ to $R_{L}$ by changing $L$ extensively $(L=9.06-135 \mathrm{~mm})$ with the bubble volume $\Omega$ fixed. All experiments are conducted for $D \ll a$ and $D \ll R_{T}$, so that $T \dot{S}_{1}$ and $T \dot{S}_{3}$ are negligibly small.

As for the coefficient $k_{2}$, there is a theoretical prediction $k_{2}=1 / 12$ under the assumption that the fluid shape is circular. This is significantly close to the value $k_{2} \cong 0.08$, confirmed in Ref. [13] for slightly elongated bubbles. The prediction was given implicitly in Ref. [19], more explicitly given in Ref. [42] through a different method, and further confirmed by experiment in Ref. [43].

\section{Bubble-shape crossover}

The crossover between the two regimes of the bubble shape from Eq. (9) to Eq. (17) is indirectly confirmed in Fig. 5(b). This figure shows $R_{T}$ as a function of the volume $\Omega$ for three different values of $L$. It reveals two regimes for $R_{T}$ : (i) For small $\Omega$, corresponding to the singly confined regime, $R_{T}$ increases with $\Omega$, in agreement with Eq. (17); (ii) For large $\Omega$, corresponding to the doubly confined regime, $R_{T}$ is constant and independent of $\Omega$, in agreement with Eq. (9). Note here that, in the singly confined regime, in which a rising bubble is not affected by side walls, $R_{T}$ scales as $r$ as announced in Eq. (17); this corresponds to the straight line observed for small $\Omega$ in Fig. 5(b).
Equation (17) means that a bubble in the singly confined regime basically keeps a circular shape $\left(R_{L} / R_{T} \cong 1\right)$; however, this is an approximate relation. In fact, when $r \gg \kappa^{-1}$, bubbles are slightly elongated and $R_{L} / R_{T}$ becomes slightly larger than one although it is not clear when plotted on a $\log$-log scale. An effect of this asymmetric factor $R_{L} / R_{T}$ is discussed in Ref [13]: although the relation $R_{L} / R_{T} \approx 1$ holds even in this previous study, the experimental data agree quite well with the velocity law in Eq. (18), which takes into account the ratio $R_{L} / R_{T}$, while the agreement clearly deteriorates when compared with Eq. (18) with setting $R_{L} / R_{T}=1$ neglecting the asymmetric effect (see Ref. [13] for the details).

\section{Phase diagram clarifying crossover}

The crossover discussed in Secs. IV B 1 and IV B 2 can be summarized in Fig. 6. The horizontal axis is the cell

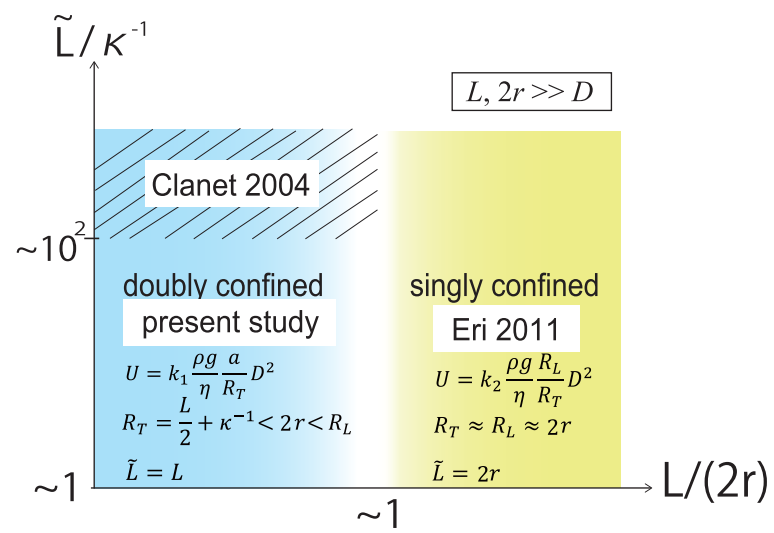

FIG. 6. Phase diagram for the dynamics of a rising bubble in the cell whose cross-section has a high aspect ratio $(L>D)$. The vertical axis is $\sqrt{B o(\tilde{L})}=\tilde{L} / \kappa^{-1}$ with the characteristic length $\tilde{L}$ (see the details for the text), while the horizontal axis is $L /(2 r)$. The doubly confined regime established in the present study [Eqs. (8)-(10)] corresponds to the left-hand side region in which $\tilde{L} / \kappa^{-1}>1$ and $L / r \ll 1$. The labels "Clanet 2004" and "Eri 2011" refer to Refs. [1] and [13], respectively. 
(a) Viscous Finger

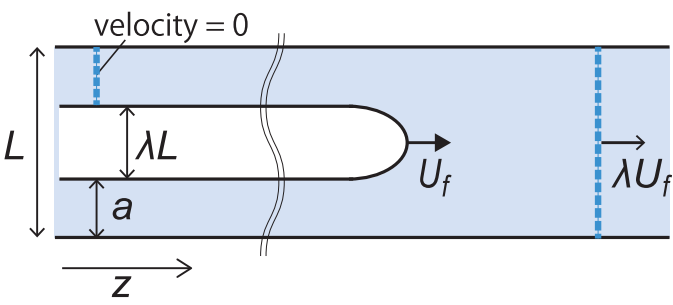

(b) Rising Bubble

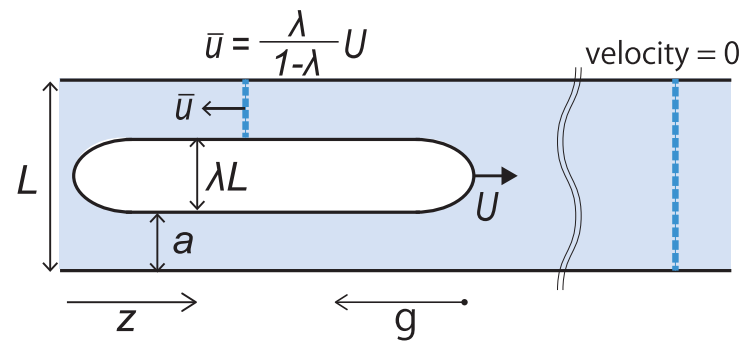

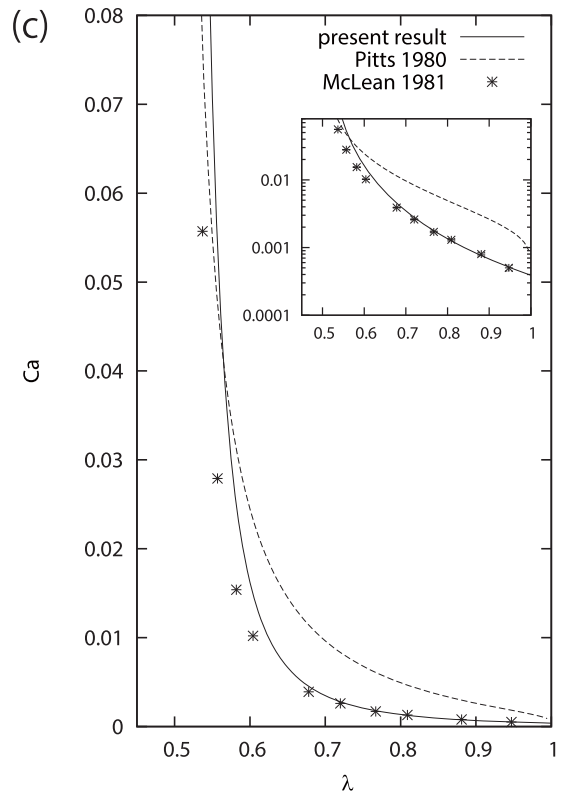

FIG. 7. [(a) and (b)] Correspondence between the two-dimensional model of viscous fingering and bubble rising in the cell thickness $D$, seen from a coordinate system fixed to the cell. The positive direction of $z$ is taken as the advancing direction of the finger or the bubble. The rising bubble is subjected to gravitational force pointing in the negative direction of $z$, while gravity is irrelevant to viscous fingering because the cell is kept horizontal. The advancing viscous finger is considered to be the same as the rising of a bubble under the transformation $U=U_{f} /(1-\lambda)$. (b) $\mathrm{Ca}\left(U_{f}\right)$ vs $\lambda$ for $D=0.08 \mathrm{~cm}$ and $L=2.54 \mathrm{~cm}$. The data labeled "Present result," "Pitts 1980," and "McLean 1981" are based on Eq. (24), Refs. [44], and [45], respectively. The curve of Pitts practically represents all the experimental data in Refs. [7,44]. The data from Ref. [45] are based on a table given in Ref. [45], and the error bars are added because some of the data have only one significant figure.

width $L$ normalized by the characteristic bubble diameter $r$ : $L /(2 r)$. The vertical axis is the square root of Bond number $\operatorname{Bo}(\tilde{L})=\rho g \tilde{L}^{2} / \gamma$ as a function of the characteristic length $\tilde{L}=L f(L /(2 r))$. Here, the dimensionless function $f(L /(2 r))$ is defined as follows:

$$
f(x)=\left\{\begin{array}{ll}
1 & (x \ll 1 ; \text { doubly confined }) \\
x^{-1} & (x \gg x ; \text { singly confined })
\end{array} .\right.
$$

In other words, $\tilde{L}$ is the smaller length scale of the two scales $L$ and $2 r: \tilde{L}=L$ for $L \ll 2 r$ (doubly confined), while $\tilde{L}=2 r$ for $L \gg 2 r$ (singly confined). As announced in Eqs. (7) and (19), the condition $\tilde{L} / \kappa^{-1} \gg 1$ is always satisfied both in the singly and doubly confined cases. With the introduction of the length scale $\tilde{L}$, we can show in Fig. 6 the doubly and singly confined regimes on the region $\tilde{L} / \kappa^{-1} \gg 1$; the doubly confined regime on the left-hand side, in which $L /(2 r) \ll 1$, and the singly confined regime on the right-hand side, in which $L /(2 r) \gg 1$. The crossover from the doubly to singly confined regime, discussed in Sec. IV B, is observed when we go from the left to the right on the phase diagram. As shown in Sec. IV A, our general formula given in Eq. (10) exhibits crossover to Eq. (14) as $\tilde{L} / \kappa^{-1}$ increases; this is observed when we go from the bottom to the top on the left-hand side of the phase diagram.

\section{CORRESPONDENCE OF BUBBLE RISING TO VISCOUS FINGERING}

Scaling laws we found in the doubly confined regime will lead to a new perspective to the study of a particular type of viscous fingering studied in Refs. [7,44,45]. Figure 7(a) illustrates the two-dimensional model of viscous fingering and rising bubble. $\lambda$ is the normalized width of finger or bubble $\left(\lambda=R_{T} / L\right)$. When the viscous finger proceeds at the speed $U_{f}$ in the coordinate system fixed to the cell, the flux in the $z$ direction (the direction in which the finger advances) averaged over the section of area $L D$ is $\lambda U_{f}$ at places far from the finger on the basis of the volume conservation $\lambda L D U_{f}=L D U(z=$ $\infty$ ), while the flux is zero in the gap (of area $a D$ ) between the bubble and the side walls. On the other hand, in the case of the bubble rising at the speed $U$, the volume conservation in Eq. (6) with $a=\left(L-R_{T}\right) / 2$ lead the downward flux $-\bar{u}=$ $-\frac{\lambda}{1-\lambda} U$ in the gap between the bubble and the side walls (of area $a D$ ) and the flux is zero at places far from the bubble. We notice that the rising bubble problem is mapped to the viscous fingering problem under the transformation:

$$
U=U_{f}(1-\lambda) .
$$

This is because, for example, if we observe the rising bubble in the reference moving at the speed $\bar{u}$ in the positive direction of $z$, the bubble tip moves at $U+\bar{u}=\frac{1}{1-\lambda} U$ and the flux far from the bubble is $\bar{u}=\frac{\lambda}{1-\lambda} U$, which are respectively mapped to $U_{f}$ and $\lambda U_{f}$ under the transformation in Eq. (22).

\section{A. Relation between relative width $\lambda$ and capillary number}

The relative width of a viscous finger $\lambda$ has been discussed as a function of the capillary number $\mathrm{Ca}\left(U_{f}\right)=\eta U_{f} / \gamma$ in the previous studies $[7,44,45]$. This issue continues to be unresolved since the pioneering work by Saffman and Taylor, i.e., more than 60 years ago, as discussed below. (In all the 
three previous, models were two-dimensional and did not take into account the existence of lubricating films of thickness $h$.)

Saffman and Taylor [7] showed experimentally that $\lambda$ was a decreasing function of $\mathrm{Ca}\left(U_{f}\right)$. In their paper, $\lambda$ approaches one as $\mathrm{Ca}\left(U_{f}\right)$ decreases while $\lambda$ approaches onehalf as $\mathrm{Ca}\left(U_{f}\right)$ increases for a cell with $D=0.08 \mathrm{~cm}$ and $L=2.54 \mathrm{~cm}$.

In 1980, Pitts [44] proposed an empirical expression that fits quite well experimental data obtained in Ref. [7] as well as obtained by himself for cells of the same geometry $(D=$ $0.08 \mathrm{~cm}$ and $L=2.54 \mathrm{~cm})$ in the range $\mathrm{Ca}\left(U_{f}\right) \simeq 0.001$ to 0.1 . He introduced an empirical assumption based on experimental observation to obtain the expression, and indicated that $\lambda$ was given as a function of $\mathrm{Ca}\left(U_{f}\right)(L / D)$ rather than of $\mathrm{Ca}\left(U_{f}\right)$ itself. The agreement between his empirical expression, shown in Fig. 7(b), and all the experimental data in Refs. [7,44] are so good that his curve in the plot can practically be considered as representing the experimental data.

In 1981, McLean and Saffman [45] proposed a numerical result indicating that $\lambda$ is given as a function of $\mathrm{Ca}\left(U_{f}\right)(L / D)^{2}$. However, their results deviate from the experimental data, represented by the Pitts curve, as shown in Fig. 7(b). Note that these deviated data points are reproduced from a table in Ref. [45], which shows a set of values of $\lambda$ and an "uncorrected version" of $\mathrm{Ca}\left(U_{f}\right)$. They also calculated "corrected" data. However, the improvement was so slight that the data labeled "McLean 1981" practically represents the two of their results.

We stress here that McLean and Saffman could not give analytical expression, as in the form $\mathrm{Ca}\left(U_{f}\right)(L / D)^{2}=F(\lambda)$. They only give a set of values $F(\lambda)$ for a discrete set of $\lambda$ via numerical calculations and the explicit functional form of $F(x)$ was not obtained in Ref. [45].

In the doubly confined regime in the present study, $\mathrm{Ca}(U)=\eta U / \gamma$ multiplied by $(L / D)^{2}$ is given explicitly as a function of $\lambda$ from Eqs. (8), (5), and (9):

$$
\mathrm{Ca}(U) \frac{L^{2}}{D^{2}} \equiv G(\lambda)=\frac{k_{1}}{2} \frac{1-\lambda}{\lambda(\lambda-1 / 2)^{2}} .
$$

Equations (22) and (23) suggest the following relation for the viscous fingering problem:

$$
\mathrm{Ca}\left(U_{f}\right) \frac{L^{2}}{D^{2}} \equiv H(\lambda)=\frac{k_{1}}{2} \frac{1}{\lambda(\lambda-1 / 2)^{2}} .
$$

In other words, the analytical expression for $F(\lambda)$, which was not available in Ref. [45] is now given as $H(\lambda)$. However, it should be noted that the function has not been determined solely by theoretical considerations. This is because this is based on the experimentally established relation Eq. (9) and the dimensionless coefficient $k_{1}$ has been determined through agreement between theory and experiment in Eq. (8).

The present result in Eq. (24) is in favor of the result of Ref. [45] in that $\lambda$ is given as a function of $\mathrm{Ca}\left(U_{f}\right)(L / D)^{2}$ rather than $\mathrm{Ca}\left(U_{f}\right)(L / D)$. In Fig. 7 (b), $\mathrm{Ca}\left(U_{f}\right)=H(\lambda)(D / L)^{2}$ is plotted for $D / L=0.08 / 2.54$. Interestingly, our prediction in Eq. (24) is in excellent agreement with the results of Ref. [45] at small $\lambda$ and with the results of the Ref. [44] at large $\lambda$. In the small $\gamma$ limit, in which $\mathrm{Ca}\left(U_{f}\right)$ diverges, $\lambda$ is predicted as $\lambda=1 / 2$, in agreement with a well-known relation for viscous fingering. However, Eq. (24) predicts $\lambda-1 / 2 \sim\left(\mathrm{Ca}\left(U_{f}\right)\right)^{-1 / 2}$ as $\mathrm{Ca}\left(U_{f}\right) \rightarrow \infty$, with a exponent $-1 / 2$, and this scaling is different form an exponent $-2 / 3$ predicted in Ref. [41]. It is worth noting that this approaches to $\lambda=1 / 2$ in the corresponding viscous fingering comes out form Eq. (9) for the bubble rising problem, which predicts that $\lambda$ approaches not $1 / 2$ but $1 / 2+\kappa^{-1} / L$.

It the context of viscous fingering, it is practically established that $\lambda$ increases toward one as $\mathrm{Ca}\left(U_{f}\right)$ decreases. This fact may be consistent with the already-mentioned wellknown relation in microfluidics $R_{T}=L-2 h$, which means $\lambda$ is close to one because $h$ is much smaller than $L$. In microfluidics, $\eta$ tends to be small and this implies $\mathrm{Ca}\left(U_{f}\right)$ tends to be small.

This consideration leads to a possibility that Eq. (9) for the bubble rising problem could be violated for significantly small $\mathrm{Ca}(U)$ because Eq. (24) does not predict $\lambda$ approaches one as $\mathrm{Ca}\left(U_{f}\right)$ decreases. To be sure, the well-known law $R_{T}=L / 2$ or $\lambda=1 / 2$ is already violated significantly when $\mathrm{Ca}\left(U_{f}\right)$ is about 0.01 and this violation is well described by Eq. (24). However, we should note that in the present study we established Eq. (9) in a limited range of $\mathrm{Ca}(U)$ approximately from 0.01 to 0.1 . This issue is an open question at the present, and it would be worth studying whether Eq. (9) could be violated for smaller $\mathrm{Ca}(U)$.

In summary, the correspondence between the present bubble rising and viscous fingering give insight for the unresolved problem concerning the viscous fingering. The corresponding previous experiments on viscous fingering have been performed not by systematically changing the ratio $D / L$ nor by carefully checking the difference between $L / 2$ and $L / 2+\kappa^{-1}$. Further experiments in this direction together with theoretical studies could be discussed in the near future.

\section{B. Shape profile of the rising bubble}

To our surprise, as shown in Fig. 8(a), we found that the shape profile near the front tip of a rising bubble scales with $R_{T}$, and that the bubble shape normalized by $R_{T}$ coincides with the shape profile of a viscous finger normalized by the finger width. Figure 8(a1) shows top shapes of the rising bubble for various $D$ and $L$. By normalizing both axes with each $R_{T}$, all shapes collapse on a single master shape as shown in Fig. 8(a2). More surprisingly, the collapsed profile of rising bubbles is the same with the normalized profile of viscous fingers. The function describing the shape profile of a viscous finger is studied both experimentally [44] and numerically (with a two-dimensional model) [45], and both agree well with experimental results. In Fig. 8(a2), the black solid line represents the shape profile of the viscous finger normalized by the finger width given in Ref. [44], showing that the shape profiles at the tip of the rising bubble and the viscous finger match almost perfectly with each other.

However, the same scenario is not applicable for the rear profile of a rising bubble. Curvature at the rear tip of a rising bubble is smaller than that at the front tip. Figure 8(b1) shows the rear shape profile for various $D$ and $L$. Different from the 

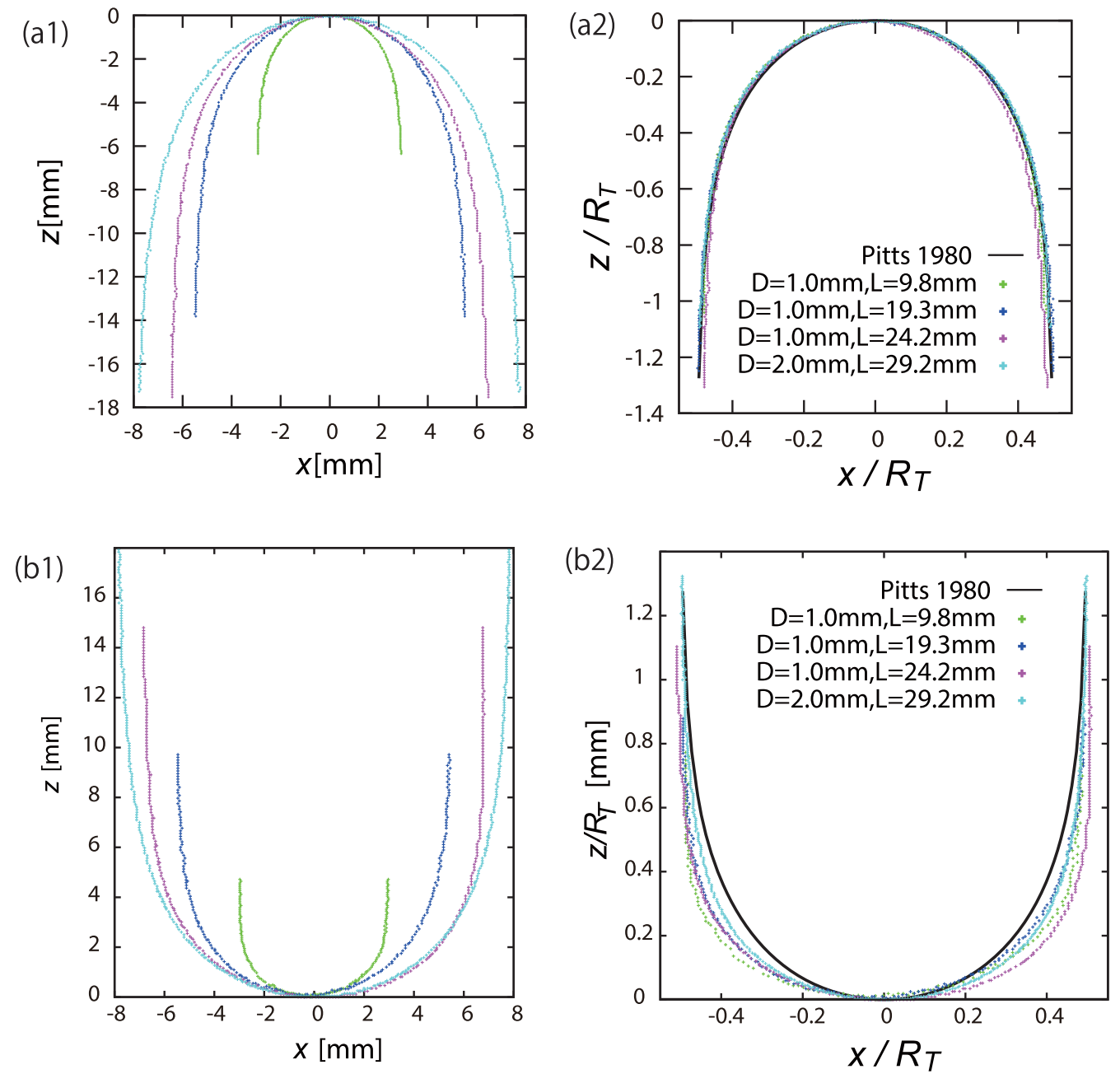

FIG. 8. (a1) Shape profiles near the front tip of bubbles in a viscous liquid of $\eta=0.48 \mathrm{~Pa}$ s. (a2) Front shapes normalized by $R_{T}$. Solid line indicates the shape of the viscous finger shown in Ref. [44]. (b1) Shape profiles near the rear tip of bubbles in a viscous liquid of $\eta=0.48$ Pa s. (b2) Rear shapes normalized by $R_{T}$. Solid line corresponds to the (inverted) shape of the viscous finger shown in (a2).

front case, the rear shapes normalized by each $R_{T}$ in both the horizontal and vertical directions do not collapse on a single master curve as shown in Fig. 8(b2). The black line indicates the shape of the viscous finger normalized by the finger width [the same one shown in Fig. 8(a2) but here with the top and bottom inverted], which shows that the curvature of the rear profile is smaller than that of the front profile.

\section{CONCLUSION}

Rising dynamics of a bubble confined in a vertical cell with a high aspect ratio rectangular cross-section was discussed, where the bubble is strongly confined in one horizontal direction and is weakly confined or is not confined in the remaining one horizontal direction. The length scales concerning the rectangular cross-section of the cell and the confined bubble involve both scales that are comparable to, and larger than, the capillary length. We focused on the case in which the inertial effect is negligible compared with the viscous effect under the influence of capillarity. We focused the doubly confined regime to find a new velocity scaling in a closed form. In deriving the velocity law in Eq. (8), we assume the conservation of the flow as in Eq. (6), which emphasizes the downward flow around the bubble (see movie 2 [40]) is essential in determining the velocity. The velocity profiles of Poiseuille flows obtained via confocal imaging on submillimeter scales were consistent with our theoretical considerations, as shown in Fig. 4. We demonstrated the crossover from this scaling regime to the singly confined regime discussed in the previous study [13]. We showed that the present doubly confined regime contains and thus reduces to the scaling regime discussed in another previous study in which capillarity is negligible [1]. These interrelationships were summarized on a phase diagram.

We also uncovered that bubble rising in the present study can be mapped to a viscous fingering [45] under a simple transformation associated with the bubble (of finger) velocity and the bubble (or finger) width. This mapping provides novel insight into the physics of viscous fingering. We showed quantitative matching of the shapes of the tips of finger and bubble, together with an analytical expression for the relative width $\lambda$ and capillary number, which has not been available in the literature since more than 60 years ago [45]. 


\section{ACKNOWLEDGMENTS}

This research was partly supported by JSPS KAKENHI (Grant Numbers JP24244066 and JP19H01859) and by the ImPACT Program of the Council for Science, Technology and Innovation (Cabinet Office, government of Japan) (Grant No.2014-PM01-02-01). M.M. is supported by a JSPS Science Research Fellowship for Young Scientists (Grant No. 16J00871). The authors thank Edward Foley (Ochanomizu University) for valuable comments on the English.
[1] C. Clanet, P. Héraud, and G. Searby, On the motion of bubbles in vertical tubes of arbitrary cross-sections: some complements to the Dumitrescu-Taylor problem, J. Fluid Mech. 519, 359 (2004).

[2] H. Wong, C. J. Radke, and S. Morris, The motion of long bubbles in polygonal capillaries. Part 1. Thin films, J. Fluid Mech. 292, 71 (1995).

[3] V. S. Ajaev and G. M. Homsy, Modeling shapes and dynamics of confined bubbles, Annu. Rev. Fluid Mech. 38, 277 (2006).

[4] P.-G. De Gennes, F. Brochard-Wyart, and D. Quéré, Capillarity and Wetting Phenomena: Drops, Bubbles, Pearls, Waves (Springer Science \& Business Media, 2013).

[5] I. Cantat, Liquid meniscus friction on a wet plate: Bubbles, lamellae, and foams, Phys. Fluids 25, 031303 (2013).

[6] P. Aussillous and D. Quéré, Bubbles creeping in a viscous liquid along a slightly inclined plane, Europhys. Lett. 59, 370 (2002).

[7] P. G. Saffman and G. Taylor, The penetration of a fluid into a porous medium or Hele-Shaw cell containing a more viscous liquid, Proc. R. Soc. London A 245, 312 (1958).

[8] F. P. Bretherton, The motion of long bubbles in tubes, J. Fluid Mech. 10, 166 (1961).

[9] S. Tanveer, The effect of surface tension on the shape of a HeleShaw cell bubble, Phys. Fluids 29, 3537 (1986).

[10] T. Maxworthy, Bubble formation, motion and interaction in a Hele-Shaw cell, J. Fluid Mech. 173, 95 (1986).

[11] A. R. Kopf-Sill and G. M. Homsy, Bubble motion in a HeleShaw cell, Phys. Fluids 31, 18 (1988).

[12] S. R. K. Maruvada and C.-W. Park, Retarded motion of bubbles in Hele-Shaw cells, Phys. Fluids 8, 3229 (1996).

[13] A. Eri and K. Okumura, Viscous drag friction acting on a fluid drop confined in between two plates, Soft Matter 7, 5648 (2011).

[14] X. Wang, B. Klaasen, J. Degrève, B. Blanpain, and F. Verhaeghe, Experimental and numerical study of buoyancydriven single bubble dynamics in a vertical Hele-Shaw cell, Phys. Fluids 26, 123303 (2014).

[15] M. Yahashi, N. Kimoto, and K. Okumura, Scaling crossover in thin-film drag dynamics of fluid drops in the Hele-Shaw cell, Sci. Rep. 6, 31395 (2016).

[16] K. Okumura, Viscous dynamics of drops and bubbles in HeleShaw cells: Drainage, drag friction, coalescence, and bursting, Adv. Colloid Interface Sci. 255, 64 (2018).

[17] L. Keiser, K. Jaafar, J. Bico, and É. Reyssat, Dynamics of nonwetting drops confined in a Hele-Shaw cell, J. Fluid Mech. 845, 245 (2018).

[18] A. Huerre, O. Theodoly, A. M. Leshansky, M.-P. Valignat, I. Cantat, and M.-C. Jullien, Droplets in Microchannels: Dynamical Properties of the Lubrication Film, Phys. Rev. Lett. 115, 064501 (2015).

[19] J. W. M. Bush, The anomalous wake accompanying bubbles rising in a thin gap: A mechanically forced Marangoni flow, J. Fluid Mech. 352, 283 (1997).
[20] M. Kawaguchi, S. Niga, N. Gou, and K. Miyake, Buoyancydriven path instabilities of bubble rising in simple and polymer solutions of Hele-Shaw cell, J. Phys. Soc. Jpn. 75, 124401 (2006).

[21] L. Zhu and F. Gallaire, A pancake droplet translating in a HeleShaw cell: Lubrication film and flow field, J. Fluid Mech. 798 , 955 (2016).

[22] J. Fabre and A. Liné, Modeling of two-phase slug flow, Annu. Rev. Fluid Mech. 24, 21 (1992).

[23] A. O. Morgado, J. M. Miranda, J. D. P. Araújo, and J. B. L. M. Campos, Review on vertical gas-liquid slug flow, Int. J. Multiphase Flow 85, 348 (2016).

[24] S. Shad, M. Salarieh, B. Maini, and I. D. Gates, The velocity and shape of convected elongated liquid drops in narrow gaps, J. Pet. Sci. Eng. 72, 67 (2010).

[25] L. J. Lee, C. Zeng, X. Cao, X. Han, J. Shen, and G. Xu, Polymer nanocomposite foams, Compos. Sci. Technol. 65, 2344 (2005).

[26] R. M. Davies and G. Taylor, The mechanics of large bubbles rising through extended liquids and through liquids in tubes, Proc. R. Soc. London, Ser. A 200, 375 (1950).

[27] E. T. White and R. H. Beardmore, The velocity of rise of single cylindrical air bubbles through liquids contained in vertical tubes, Chem. Eng. Sci. 17, 351 (1962).

[28] K. W. Tung and J.-Y. Parlange, Note on the motion of long bubbles in closed tubes-influence of surface tension, Acta Mech. 24, 313 (1976).

[29] E. E. Zukoski, Influence of viscosity, surface tension, and inclination angle on motion of long bubbles in closed tubes, J. Fluid Mech. 25, 821 (1966).

[30] H. L. Goldsmith and S. G. Mason, The movement of single large bubbles in closed vertical tubes, J. Fluid Mech. 14, 42 (1962).

[31] T. M. Squires and S. R. Quake, Microfluidics: Fluid physics at the nanoliter scale, Rev. Mod. Phys. 77, 977 (2005).

[32] G. M. Whitesides, The origins and the future of microfluidics, Nature (London) 442, 368 (2006).

[33] M. Sadatomi, Y. Sato, and S. Saruwatari, Two-phase flow in vertical noncircular channels, Int. J. Multiphase Flow 8, 641 (1982).

[34] K. Mishima, T. Hibiki, and H. Nishihara, Some characteristics of gas-liquid flow in narrow rectangular ducts, Int. J. Multiphase Flow 19, 115 (1993).

[35] Q. C. Bi and T. S. Zhao, Taylor bubbles in miniaturized circular and noncircular channels, Int. J. Multiphase Flow 27, 561 (2001).

[36] S. Bhusan, S. Ghosh, G. Das, and P. K. Das, Rise of Taylor bubbles through narrow rectangular channels, Chem. Eng. J. 155, 326 (2009).

[37] W. B. Kolb and R. L. Cerro, Coating the inside of a capillary of square cross section, Chem. Eng. Sci. 46, 2181 (1991). 
[38] J. Bico and D. Quéré, Rise of liquids and bubbles in angular capillary tubes, J. Colloid Interface Sci. 247, 162 (2002).

[39] Q. Liao and T. S. Zhao, Modeling of Taylor bubble rising in a vertical mini noncircular channel filled with a stagnant liquid, Int. J. Multiphase Flow 29, 411 (2003).

[40] See Supplemental Material at http://link.aps.org/supplemental/ 10.1103/PhysRevResearch.2.013188 for movies.

[41] R. Combescot, T. Dombre, V. Hakim, Y. Pomeau, and A. Pumir, Shape Selection of Saffman-Taylor Fingers, Phys. Rev. Lett. 56, 2036 (1986).
[42] F. Gallaire, P. Meliga, P. Laure, and C. N. Baroud, Marangoni induced force on a drop in a Hele Shaw cell, Phys. Fluids 26, 062105 (2014).

[43] I. Shukla, N. Kofman, G. Balestra, L. Zhu, and F. Gallaire, Film thickness distribution in gravity-driven pancake-shaped droplets rising in a Hele-Shaw cell, J. Fluid Mech. 874, 1021 (2019).

[44] E. Pitts, Penetration of fluid into a Hele-Shaw cell: The Saffman-Taylor experiment, J. Fluid Mech. 97, 53 (1980).

[45] J. W. McLean and P. G. Saffman, The effect of surface tension on the shape of fingers in a Hele Shaw cell, J. Fluid Mech. 102, 455 (1981). 